\title{
Lipoprotein-associated phospholipase A2 in relation to lipid markers: A pan-India correlation study
}

\author{
Sandhya lyer, Rajkumar Kushawaha, Prachi Sinkar, Sivakumar Selvaraj, \\ Amruta Velumani \\ Biochemistry Division, Thyrocare Technologies Limited, Plot No.D37/1, TTC Industrial area, MIDC, Turbhe, \\ Navi Mumbai - 400703, India
}

Background: Conventional serum markers are routinely recommended to assess heart health. However, many new biochemical markers like lipoprotein-associated phospholipase A2 (Lp-PLA2) has been studied as an efficient cardiac risk marker. Aims and Objective: The current study was designed to link the correlation between traditional lipid markers and Lp-PLA2 in a pan-India cohort. Materials and Methods: The study cohort included a total of 10347 pan-Indians including 5945 males and 4402 females respectively. The serum lipid parameters estimated include triglycerides, LDL- and HDL-cholesterol as well as total cholesterol by the technology of photometry. Results: The frequency of high Lp-PLA2 detected in our study was $6.4 \%$ and the frequency between males at $9.3 \%$ and females at $2.5 \%$ was detected to be significant at $p<0.0001$. Linear regression analysis detected significant positive association of high Lp-PLA2 with LDL-cholesterol and total cholesterol at $p<0.05$, while the trend with HDL-cholesterol was also significant. However, Lp-PLA2 as an independent marker was not detected to be significant in relation to the lipid indices studied. Conclusion: Lp-PLA2 has been associated with risk factor for acute coronary crisis; its clinical value remains controversial. Our report though detected significant association of Lp-PLA2 with various lipid parameters, independent value remains questionable.

Key words : Lp-PLA2; Cardiac risk; Cholesterol; India; LDL; Triglycerides
http://nepjol.info/index.php/AJMS DOI: 10.3126/ajms.v10i3.22791 E-ISSN: 2091-0576 P-ISSN: 2467-9100

\section{INTRODUCTION}

Lipoprotein-associated phospholipase A2 (Lp-PLA2) is an enzyme associated with low-density lipoproteins (LDL) and is involved in the hydrolysis of phospholipids. Studies have suggested Lp-PLA2 to have a strong proinflammatory role and thus is a key contributing factor for atherosclerosis, plaque stability as well as severity of coronary artery stenosis. ${ }^{1,2}$ The clinical utility of Lp-PLA2 continues to be controversial as it has been proven to play a role in progression of atherosclerosis on one line while clinical trials involving Lp-PLA2 inhibitors have not shown significant favorable outcomes. ${ }^{3,4}$ Lp-PLA2 has been widely proposed in the clinical context to be used for risk stratification. ${ }^{1}$ Lp-PLA2 is produced by the macrophages as well as lymphocytes which bind to LDL and oxidizes the same to produce free fatty acids and lyolecithin. ${ }^{5}$

Many cardiac markers are utilized for routine screening like cardiac troponin specifically cardiac troponin I (cTn I) for acute myocardial infarction. However, the sensitivity has been proven to be low because of its association with many other conditions like renal insufficiency, myocarditis, heart failure, etc. ${ }^{6}$ In contrast, Lp-PLA2 comparison studies with traditional inflammatory and myocardial injury markers detected the former to bear higher diagnostic value pertaining to early diagnosis. ${ }^{2}$ The evidence of positive association has been proven in many ethnic groups with acute myocardial infarction, cardiovascular disease (CVD) as well as incidence of coronary heart disease. ${ }^{7,8}$ LDLs are 
the primary target of cholesterol-lowering therapy though many studies have highlighted its atherogenic predictive strength to be good only on a population basis and weak when it comes to individual cases. ${ }^{1}$ Triglycerides (TG) and their role in cardiovascular risk continue to be controversial as studies have shown elevated levels of the same to also be due to metabolic syndrome or insulin resistance. ${ }^{9}$ Though the biochemical structure of TG limits its ability to directly cause any atherosclerotic CVD, a mild-to-moderate raise in levels have been shown to bear the potential to trigger the inflammation cascade which can promote atherosclerosis. ${ }^{10}$ Total cholesterol (TC) has been positively associated as a strong risk factor for coronary heart disease with the effect being slightly strong for men as compared to women. ${ }^{11}$ Studies have also shown a strong inverse correlation to exist between levels of high-density lipoprotein (HDL) cholesterol and coronary disease, with outcomes of Framingham study highlighting increase in HDL-C by $10 \mathrm{mg} / \mathrm{L}$ to cause risk reduction of up to $2-3 \%{ }^{12}$

Lp-PLA2 has been reported to be a good predictor of cardiac disease by many studies. A review study based on outcomes reported by 14 observational studies involving over 20,000 participants detected the predictive ability of Lp-PLA2 at combined odds of 1.60 (95\% CI, 1.36 to 1.89) towards risk of future adverse cardiac events. ${ }^{13}$ Association studies have also reported on correlation of Lp-PLA2 with different parameters of CVD. One such study which evaluated the association between Lp-PLA2 and the protective effect of HDL-C on 3888 CVD adults detected the relation between HDL-C levels and risk of incident coronary heart disease to be attenuated among patients in the highest tertile of Lp-PLA2. However, this study did report on absence of consistent association between high Lp-PLA2 and risk across different HDL-C categories. ${ }^{14}$ Another Nature scientific report which studied Lp-PLA2 and oxidized-LDL among young Chinese patients with acute coronary syndrome detected significant correlation between Lp-PLA2 levels and smoking, TC, LDL-C as well as oxidized LDL levels at significance $\mathrm{p}<0.05$. This study concluded Lp-PLA2 to be a marker of oxidative stress and inflammation than an independent risk factor for acute coronary syndrome among young Chinese. ${ }^{15}$

Based on this background, our study was aimed at evaluating the association and trend between Lp-PLA2 and traditional lipid parameters like triglycerides, LDL and HDL cholesterol as well as total cholesterol in a panIndia cohort.

\section{MATERIALS AND METHODS}

\section{Cohort}

This retrospective analysis included data from 10,347 inclusive of 5945 males and 4402 females respectively. The need for specific informed consent was deviated from, for this retrospective analysis involved use of no patient identifiers for analysis or drawing conclusion apart from gender and testing was done in our clinical reference laboratory and not hospital-based. The entire cohort characteristics have been highlighted in Table 1.

\section{Estimation of Lp-PLA2 and lipid parameters}

Serum Lp-PLA2 estimation was done using the Diazyme Lp-PLA2 enzymatic assay in photometry technology (Olympus AU400, Beckman Olympus). The test involved hydrolysis of the substrate 1-myristoyl-2-(4nitrophenylsuccinyl) phosphatidylcholine at the sn-2 position producing 4-nitrophenol, a colored product measured spectrophotometrically at $405 \mathrm{~nm}$. The activity was calculated from the rate of change in absorbance. The critical call values were set at $275 \mathrm{U} / \mathrm{L}$ wherein any value below this was reported to be low risk and above as high risk.

Estimation for lipid parameters like TG, LDL-C, HDL-C and TC was done using dedicated enzymatic assay kits from Sekure Chemistry (Sekisui Diagnostics, UK) and estimated on photometry technology (Olympus AU2700, Beckman Olympus) as per manufacturer instructions. The quality control measures involved use of two-level serum controls from BioRad Laboratories (Hercules, CA). Trend analysis was done using LJ plots and compliance with Westguard rule was evaluated. The reference ranges for reporting were as follows; TG - $25-200 \mathrm{mg} / \mathrm{dL}$; LDL-C - 85 - $130 \mathrm{mg} / \mathrm{dL}$; Cholesterol - 125 - $200 \mathrm{mg} / \mathrm{dL}$; HDL-C - $35-80 \mathrm{mg} / \mathrm{dL}$.

\section{RESULTS}

\section{Cohort analysis}

Out study analysis included data from a total of 10347 individuals including 5945 males (46 +/- 14 years) and 4402 females ( $46+/-14$ years) studied to assess relation and

\section{Table 1: Study cohort characteristics}

\begin{tabular}{lcccccc}
\hline Cohort & $\mathbf{N}$ & $\begin{array}{c}\text { Mean age } \\
\text { (Years) }\end{array}$ & $\begin{array}{c}\text { Mean TG } \\
\text { (mg/dL) }\end{array}$ & $\begin{array}{c}\text { Mean LDL-C } \\
\text { (mg/dL) }\end{array}$ & $\begin{array}{c}\text { Mean total } \\
\text { cholesterol (mg/dL) }\end{array}$ & $\begin{array}{c}\text { Mean } \\
\text { Lp-PLA2 (U/L) }\end{array}$ \\
\hline Males & 5945 & $46 \pm 14$ & $157 \pm 95$ & $116 \pm 34$ & $185 \pm 42$ & $200 \pm 57$ \\
Females & 4402 & $46 \pm 14$ & $125 \pm 74$ & $114 \pm 32$ & $184 \pm 39$ & $167 \pm 48$ \\
Total & 10347 & $46 \pm 14$ & $143 \pm 88$ & $115 \pm 33$ & $184 \pm 41$ & $180 \pm 11$ \\
\hline
\end{tabular}


trend between Lp-PLA2 and lipid parameters like TG, TC, LDL-C and HDL-C. The average levels of each of the lipid parameter studied has been highlighted already in Table 1.

Trend analysis between Lp-PLA2 and individual lipid parameters highlighted a positive correlation. Multiple regression analysis highlighted significance of Lp-PLA2 in relation to all lipid parameters at $\mathrm{p}<0.01$. The same has been highlighted in Figureure 1(a), 1(b), 1(c), 1(d).

Further analysis was also done to detect if a trend exists between normal levels of lipid parameters in relation to high Lp-PLA2 levels. No significant trend was observed when the probability of this association was put to test. The detailed analysis and outcome has been highlighted in Table 2.

The difference in frequency between males and females in all the cases was significant at $\mathrm{p}<0.0001$ by Chi-Square with Yates correction.
High Lp-PLA2 analysis outcome

Analysis of high Lp-PLA2 values in relation to different lipid indices was also studied and highlighted in Figure 2.

Summary of frequency as well as average serum levels have been detailed in Table 3. The difference in frequency between males and females was found to be significant at $\mathrm{p}<0.0001$ by Chi-Square test with Yates correction.

Linear regression analysis was also done to detect significance of association between each lipid parameter with high Lp-PLA2 levels. Significance was detected for LDL-C F $(1,661)=13.9, \mathrm{p}<0.05$, for TC F $(1,661)=12.1$, $\mathrm{p}<0.05$ and for HDL-C at $\mathrm{F}(1,661)=4.6, \mathrm{p}<0.05$. No significance was detected for TG with high Lp-PLA2. However when considering the strength of high Lp-PLA2 as an independent risk factor, the odds were detected to be 0.5 (95\%CI, $0.4-0.6)$ for TG, 0.3 (95\% $\%$ CI, $0.3-0.4)$ for LDL-C and 0.3 (95\%CI, $0.2-0.4)$ for TC. Further, the frequency of individuals with high values for Lp-PLA2

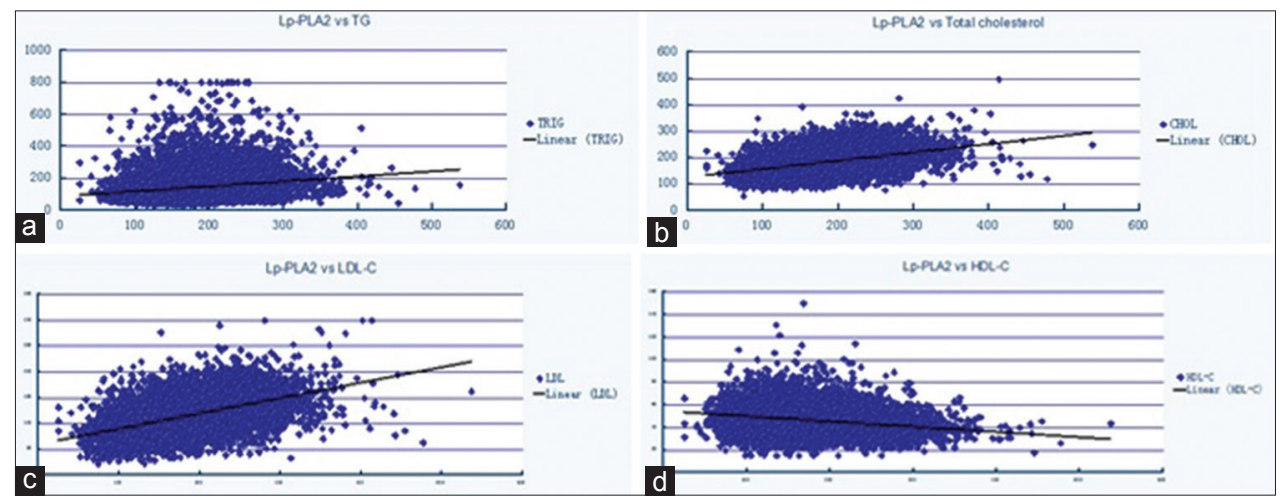

Figure 1: (a) Trend of Lp-PLA2 vs TG (b) Trend of Lp-PLA2 vs Total cholesterol (c) Trend of Lp-PLA2 vs LDL-C (d) Trend of Lp-PLA2 vs HDL-C

Table 2: Frequency analysis outcome of normal values of lipid indices vs. high Lp-PLA2

\begin{tabular}{|c|c|c|c|c|c|}
\hline Cohort & Normal TG (N) & $\begin{array}{c}\text { High } \\
\text { Lp-PLA2 (N) }\end{array}$ & $\begin{array}{c}\text { Frequency of } \\
\text { high Lp-PLA2 (\%) }\end{array}$ & $\begin{array}{c}\text { Mean TRIG levels } \\
\text { (mg/dL) }\end{array}$ & $\begin{array}{c}\text { Mean Lp-PLA2 } \\
\text { levels (U/L) }\end{array}$ \\
\hline Males & 4645 & 381 & 8.2 & $119 \pm 40$ & $196 \pm 56$ \\
\hline Females & 3900 & 82 & 2.1 & $105 \pm 39$ & $165 \pm 47$ \\
\hline Total & 8545 & 463 & 5.4 & $113 \pm 40$ & $182 \pm 55$ \\
\hline Cohort & $\begin{array}{l}\text { Normal } \\
\text { LDL-C (N) }\end{array}$ & $\begin{array}{c}\text { High } \\
\text { Lp-PLA2 (N) }\end{array}$ & $\begin{array}{c}\text { Frequency of } \\
\text { high Lp-PLA2 (\%) }\end{array}$ & $\begin{array}{l}\text { Mean LDL-C } \\
\text { levels (mg/dL) }\end{array}$ & $\begin{array}{c}\text { Mean Lp-PLA2 } \\
\text { levels (U/L) }\end{array}$ \\
\hline Males & 2848 & 184 & 6.5 & $109 \pm 13$ & $197 \pm 49$ \\
\hline Females & 2313 & 39 & 1.7 & $108 \pm 13$ & $164 \pm 43$ \\
\hline Total & 5161 & 223 & 4.3 & $109 \pm 13$ & $182 \pm 49$ \\
\hline Cohort & $\begin{array}{c}\text { Normal total } \\
\text { cholesterol (N) }\end{array}$ & $\begin{array}{c}\text { High } \\
\text { Lp-PLA2 (N) }\end{array}$ & $\begin{array}{c}\text { Frequency of } \\
\text { high Lp-PLA2 (\%) }\end{array}$ & $\begin{array}{l}\text { Mean total cholesterol } \\
\text { levels (mg/dL) }\end{array}$ & $\begin{array}{c}\text { Mean Lp-PLA2 } \\
\text { levels (U/L) }\end{array}$ \\
\hline Males & 3461 & 203 & 5.9 & $168 \pm 21$ & $191 \pm 52$ \\
\hline Females & 2772 & 38 & 1.4 & $167 \pm 20$ & $158 \pm 44$ \\
\hline Total & 6233 & 241 & 3.9 & $167 \pm 20$ & $176 \pm 52$ \\
\hline Males & 4742 & 395 & 8.3 & $46 \pm 8$ & $199 \pm 55$ \\
\hline Females & 4050 & 88 & 2.2 & $52 \pm 10$ & $166 \pm 47$ \\
\hline Total & 8792 & 483 & 5.5 & $48 \pm 9$ & $184 \pm 54$ \\
\hline
\end{tabular}




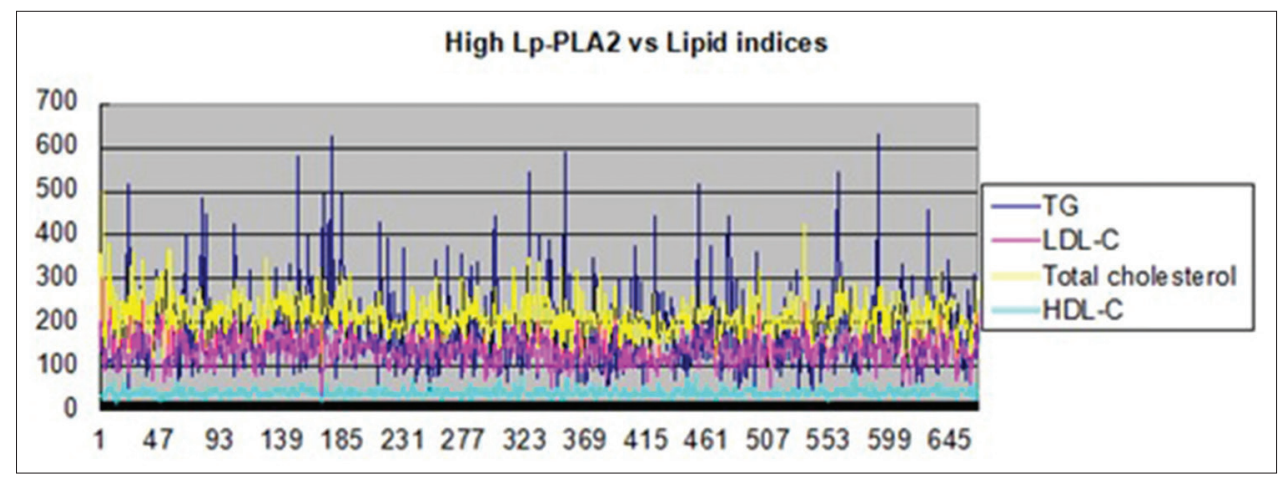

Figure 2: Analysis of high Lp-PLA2 in relation to estimated lipid indices

\begin{tabular}{lccccccc}
\multicolumn{6}{l}{ Table 3: High Lp-PLA2 (>275 U/L) analysis outcome } \\
Cohort & $\mathbf{N}$ & Frequency (\%) & $\begin{array}{c}\text { Mean } \\
\text { Lp-PLA2 (U/L) }\end{array}$ & $\begin{array}{c}\text { Mean TG } \\
\text { (mg/dL) }\end{array}$ & $\begin{array}{c}\text { Mean LDL-C } \\
\text { (mg/dL) }\end{array}$ & $\begin{array}{c}\text { Mean total } \\
\text { cholesterol (mg/dL) }\end{array}$ & $\begin{array}{c}\text { Mean HDL-C } \\
\text { (mg/dL) }\end{array}$ \\
\hline Males & 555 & 9.3 & $303 \pm 30$ & $182 \pm 95$ & $142 \pm 35$ & $214 \pm 45$ & $40 \pm 9$ \\
Females & 108 & 2.5 & $308 \pm 35$ & $168 \pm 74$ & $141 \pm 37$ & $213 \pm 50$ & $42 \pm 9$ \\
Total & 663 & 6.4 & $304 \pm 31$ & $179 \pm 92$ & $142 \pm 35$ & $214 \pm 46$ & $40 \pm 9$ \\
\hline
\end{tabular}

and all the assessed lipid parameters like TG, LDL-C and TC was detected to be $1.1 \%$ inclusive of males at $1.6 \%$ and females at $0.4 \%$ and the difference was statistically significant at $\mathrm{p}<0.0001$ by Chi-Square test with Yates correction.

\section{DISCUSSION}

Our study report aimed at determining the correlation between Lp-PLA2 and lipid parameters to identify the efficacy of the same as a good cardiac risk marker. Though the association of Lp-PLA2 as a strong risk predictor is still controversial, our aim was to identify trend association between Lp-PLA2 and conventional lipid markers.

Lp-PLA2 is secreted by the inflammatory cells in the human body and the majority $\sim 80 \%$ is bound to lipoproteins like LDL and to a lesser extent to HDL at roughly 10\%. Many studies have identified elevated Lp-PLA2 activity to be associated with risk of CVD. ${ }^{16}$ Numerous studies have analyzed the strength of Lp-PLA2 as an independent risk marker in comparison with traditional cardiac risk factors and one such study which analyzed a total of 20,549 patients detected the combined odds to be $1.60(95 \% \mathrm{CI}, 1.36$ - 1.89) towards development of future cardiac effects. ${ }^{13}$ Another meta-analysis which reported on evaluation of Lp-PLA2 levels with stroke, CAD and mortality from a total of 79,036 participants detected significance with all three factors at relative risk of 1.08 (95\% CI, 0.97 - 1.20) for stroke, 1.10 (95\% CI, 1.04 1.17) for CAD and 1.16 (95\% CI, 1.09 - 1.24) for vascular mortality. ${ }^{17}$ However, our study which analyzed Lp-PLA2 specifically in relation to lipid parameters detected no such association when frequency of high Lp-PLA2 levels was compared with normal levels of lipid indices as well as with high levels of lipids. Thus, Lp-PLA2 in comparison to traditional lipid parameters like TG, TC and LDL-C was not detected to have a significant impact towards cardiac risk independently.

A study by the Atherosclerosis Risk in Communities (ARIC) examined the relation between two inflammatory markers; Lp-PLA2 and high-sensitivity C-reactive protein (CRP) in a case-control manner. This study detected high levels of Lp-PLA2 in incident cases of coronary heart disease and also among individuals with non-elevated levels of LDL. Lp-PLA2 was detected to be independently associated with risk post adjustment for CRP and traditional risk factors. ${ }^{18}$ In our study, the detected frequency of high Lp-PLA2 was $4.3 \%$ in the cohort with non-elevated LDL levels and males were found to be higher at $6.5 \%$ in comparison to females at $1.7 \%$, significant at $\mathrm{p}<0.0001$. Though linear regression analysis detected significance of high Lp-PLA2 in relation to LDL levels, the strength of Lp-PLA2 independently was detected to be weak. One probability could be that our analysis did not take into account other traditional cardiac risk factors into consideration like BMI, blood pressure, smoking status, metabolic syndrome, etc.

A recent study among patients with acute coronary syndrome from China included 228 young patients matched with 237 control subjects and found significant correlation between Lp-PLA2 levels and smoking status, TC, LDL as well as oxidized LDL at $\mathrm{p}<0.05$. Further multivariate logistic regression detected oxidized LDL, CRP, TG, smoking and male gender to be independently associated with risk of acute coronary syndrome. However, 
analysis post adjustment for oxidized-LDL, detected the association between Lp-PLA2 and acute coronary syndrome to become insignificant, indicating the risk to be dependent on levels of the later. Lack of improvement in risk prediction detected Lp-PLA2 to be a better marker of oxidative stress than CVD. ${ }^{15}$ Our study detected high levels of Lp-PLA2 among individuals with normal levels of lipids, thus highlighting the risk of oxidative stress to be present irrespective of lipid levels. In our study the frequency of high lipid markers with high Lp-PLA2 levels were detected to be $1.1 \%$ with the frequency among males to be higher at $1.6 \%$ compared to females at $0.4 \%$ and significant at $\mathrm{p}<0.0001$.

Many Indian reports have also studied the efficacy of Lp-PLA2 as an emerging cardiovascular marker. One such cross-sectional study evaluated a total of 391 South Indian subjects including 271 test subjects and 120 controls. This study detected test subjects to have significantly high levels of TG, LDL, TC, random blood sugar and BP in comparison to controls at $\mathrm{p}<0.001$. The mean Lp-PLA2 levels were also detected to be high at $565.50 \mathrm{~g} / \mathrm{L}$ in the test group in comparison to controls at $395.07 \mathrm{~g} / \mathrm{L} .{ }^{19} \mathrm{In}$ our study, subjects with high Lp-PLA2 were detected to harbor borderline levels of high TG at $179+/-92 \mathrm{mg} / \mathrm{dL}$, high LDL-C at $142+/-35 \mathrm{mg} / \mathrm{dL}$ and TC at $214+/-46 \mathrm{mg} / \mathrm{dL}$ respectively. Significance with LDL-C and $\mathrm{TC}$ was detected by linear regression analysis at $\mathrm{p}<0.05$. Another report studied association between Lp-PLA2, oxidized LDL and glycaemic status in early stages of type 2 diabetes including 40 individuals each of case and control. This study detected significantly higher levels of the same in cases as compared to controls and a positive correlation with oxidized LDL among cases $(\mathrm{r}=0.542, \mathrm{P}<0.01)$ and controls $(\mathrm{r}=0.414, \mathrm{P}<0.01)$, thus proving activity of LpPLA2 to be highly affected by oxidized LDL. ${ }^{8}$ Another Indian report which studied Lp-PLA2 and carotid intima media thickness (CIMT) as markers of increased CVD risk among 70 subjects each in case and controls, detected no relationship between predicting CIMT and Lp-PLA2 activity. ${ }^{20}$

\section{CONCLUSION}

The significance of Lp-PLA2 as an independent cardiac risk marker continues to be studied in comparison to many biochemical markers. Highlighting a single association with proven end-point of improved patient outcome needs more clinical trial studies in large population size. Patient management using Lp-PLA2 levels and its monitoring is not currently a recommendation. Though the background for evidence remains controversial, our study does show a positive trend between high levels of Lp-PLA2 with total cholesterol, LDL-C as well as HDL-C and hence these can be monitored together for patient management.

\section{ETHICAL APPROVAL}

Not required. This study involved data analysis from a reference laboratory and only details pertaining to gender and age was taken for analysis. No other patient identifiers were noted or mentioned in the manuscript to the extent of compromising patient confidentiality.

\section{ACKNOWLEDGEMENT}

None.

\section{REFERENCES}

1. Medical Policy. Measurement of lipoprotein-associated phospholipase A2 in the assessment of cardiovascular risk. BlueCross BlueShield, Kansas, 2018.

2. Caslake MJ, Packard CJ, Robertson M, Cooney J, Nelson JJ, Ford I, et al. Lipoprotein-associated phospholipase $\mathrm{A}(2)$, inflammatory biomarkers, and risk of cardiovascular disease in the Prospective Study of Pravastatin in the Elderly at Risk (PROSPER). Atherosclerosis 2010; 210(1): 28 - 34.

3. Nicholls SJ, Kastelein JJ, Schwartz GG, Bash D, Rosenson RS, Cavender MA, et al. Varespladib and cardiovascular events in patients with an acute coronary syndrome: the VISTA-16 randomized clinical trial. JAMA 2014; 311(3): $252-262$

4. O'Donoghue ML, Braunwaid E, White HD, Lukas MA, Tarka E, Steg PG, et al. Effect of darapladib on major coronary events after an acute coronary syndrome: the SOLID-TIMI 52 randomized clinical trial. JAMA 2014; 312(10): 1006 - 1015.

5. Wu X, Zhang Y, Wu Z, You W, Liang F, Ye F, et al. Plasma lipoprotein-associated phospholipase A2 level is an independent predictor of high thrombus burden in patients with acute STsegment elevation myocardial infarction. Int Heart J 2016; 57(6): $689-696$.

6. Cardinaels EP, Altintas S, Versteylen MO, Joosen IA, Jellema LJ, Wildberger JE, et al. High-sensitivity cardiac troponin concentrations in patients with chest discomfort: Is it the heart or kidneys as well? PLoS One 2016; 11(4): e0153300.

7. Sun T, Zhao Q, Yin Z, Xu Z, Zhuo Y, Fan Li, et al. Lipoproteinassociated phospholipase A2 activity level may be complementary to cardiactroponin I as a biomarker for acute myocardial infarction in Chinese patients with chest pain. J Biomed 2018; 3: 19 - 25.

8. Garg S, Madhu SV and Suneja S. Lipoprotein associated phospholipase A2 activity \& its correlation with oxidized LDL \& glycaemic status in early stages of type- 2 diabetes mellitus. Ind J Med Res 2015; 141(1): 107 - 114.

9. West KM, Ahuja MM, Bennett PH, Czyzyk A, De Acosta OM, Fuller $\mathrm{JH}$, et al. The role of circulating glucose and triglyceride concentrations and their interactions with other "risk factors" as determinants of arterial disease in nine diabetic population samples from the WHO multinational study. Diab Care 1983; 6(4): $361-369$.

10. Shaikh M, Wootton R, Nordestgaard BG, Baskerville $P$, Lumley JS, La Ville AE, et al. Quantitative studies of transfer in vivo of low density, Sf $12-60$, and Sf $60-400$ lipoproteins 
between plasma and arterial intima in humans. Arterioscler Thromb 1991; 11(3): $569-577$.

11. Peters SA, Singhateh $Y$, Mackay D, Huxley RR and Woodward M Total cholesterol as a risk factor for coronary heart disease and stroke in women compared with men: A systematic review and meta-analysis. Atherosclerosis 2016; 248: 123 - 131.

12. Ali KM, Wonnerth $A$, Huber $K$ and Wojta J. Cardiovascular disease risk reduction by raising HDL cholesterol - current therapies and future opportunities. Br J Pharmacol 2012; 167(6): 1177 - 1194.

13. Garza CA, Montori VM, McConnell JP, Somers VK, Kullo IJ and Lopez-Jimenez F. Association between lipoprotein-associated phospholipase A2 and cardiovascular disease: a systematic review. Mayo Clin Proc 2007; 82(2): 159 - 165.

14. Tehrani DM, Gardin JM, David Y, Hirsch CH, Lloyd-Jones DM, Stein PK, et al. Impact of inflammatory biomarkers on relation of high density lipoprotein cholesterol with incident coronary heart disease: Cardiovascular Health Study. Atherosclerosis 2013; 231(2). DOI: 10.1016/j.atherosclerosis.2013.08.036

15. Huang Y, Wu Y, Yang Y, Li W and Hu Y. Lipoprotein-associated phospholipase A2 and oxidized low-density lipoprotein in young patients with acute coronary syndrome in China. Scientific Reports 2017; 7: 16092.
16. Vittos $\mathrm{O}$, Toana $\mathrm{B}$, Vittos $\mathrm{A}$ andMoldoveanu $\mathrm{E}$. Lipoproteinassociated phospholipase A2 (Lp-PLA2): a review of its role and significance as a cardiovascular marker. Biomarkers 2012; 17(4): $289-302$.

17. Lp-PLA(2) Studies Collaboration, Thompson A, Gao P, Orfei L, Watson S, Di Angelantonio E, et al. Lipoproteinassociated phospholipase $\mathrm{A}(2)$ and risk of coronary disease, stroke and mortality: collaborative analysis of 32 prospective studies. Lancet 2010; 375(9725): 1536 - 1544.

18. Ballantyne $\mathrm{CM}$, Hoogeveen RC, Bang $\mathrm{H}$, Coresh J, Folsom AR, Heiss G, et al. Lipoprotein-associated phospholipase A2, highsensitivity C-reactive protein, and risk for incident coronary heart disease in middle-aged men and women in the Atherosclerosis Risk in Communities (ARIC) study. Circulation 2004; 109(7): $837-842$.

19. Mirshad PV, Jithesh TK, Mahendra J andGurumurthy P. Lipoprotein associated Phospholipase A2 (Lp-PLA2) as an emerging cardiovascular marker. Am J Biochem 2017; 7(3): 47 - 53.

20. Kumar D, Mishra AK, Siddiqui MS andQureshi A. Study of lipoprotein-associated phospholipase A2 and carotid intima thickness as markers of increased cardiovascular risk. Int J Contemp Med Res 2017; 4(5): 1083 - 1085.

\section{Authors Contribution:}

SI- Concept and Study design, Analysis and Interpretation of data and manuscript preparation; RK- Data acquisition; PS- Concept and study design, Analysis and interpretation of data, Revision of manuscript; SS- Concept and study design, drafting of manuscript; AV- Data acquisition, revision of manuscript.

Work attributed to:

Department of Biochemistry, Thyrocare Technologies Limited

Orcid ID:

Dr. Sandhya lyer - https://orcid.org/0000-0002-8872-0322

Mr. Rajkumar Kushawaha - (i) https://orcid.org/0000-0001-5646-2229

Dr. Prachi Sinkar - https://orcid.org/0000-0002-3336-5140

Dr. Sivakumar Selvaraj - (D https://orcid org/0000-0002-6912-1906

Ms. Amruta Velumani - (D https://orcid.org/0000-0002-8337-247X

Source of Support: Nil, Conflict of Interest: None declared. 